\title{
RAIL DETECTION USING LIDAR SENSORS
}

\author{
D. STEIN, M. SPINDLER, J. KUPER \& M. LAUER \\ Institute of Measurement and Control Systems, Karlsruhe Institute of Technology, Germany.
}

\begin{abstract}
This article investigates in which way a lidar sensor can be used in a train-borne localization system. The idea is to sense infrastructure elements like rails and turnouts with the lidar sensor and to recognize those objects with a template-matching approach. A requirement analysis for the lidar sensor is presented and a market review based on these requirements is performed. Furthermore, an approach for template matching on lidar scans to recognize infrastructure objects is introduced and its empirical performance is demonstrated based on measurements taken in a light rail environment.

The overall goal of the integration of lidar sensors is to fill the sensory gap of existing train localization approaches, which are able to determine the exact, track-selective train position only if highly accurate position measurements from satellite navigation systems are available, which is often not the case. By integrating a lidar sensor, the localization system becomes more diverse, more robust, and can tolerate missing or faulty measurements from the satellite navigation system.
\end{abstract}

Keywords: lidar sensor, rail detection, train-borne localization.

\section{INTRODUCTION}

Automatic train protection systems are designed to preclude that two trains are on the same track segment at the same time. Therefore, train protection systems have to be able to determine the position of all trains accurately and reliably. Present state-of-the-art systems are mainly based on infrastructure elements, that is, axle counters and balises, to determine which track segments are occupied and which ones are free [1]. Some modern systems like the German Linienzugbeeinflussung (LZB) or the European Train Control System (ETCS) [2] also use velocity sensors on the trains to update the train position continuously in time between two balises. However, even those systems are highly dependent on infrastructure elements.

Although infrastructure-based train localization systems have a long tradition, they suffer from two facts. First of all, infrastructure elements are expensive and they are object of vandalism and theft. Therefore, future train localization systems should require a minimum of infrastructure elements. Secondly, the throughput of a track, that is, the number of trains that can be operated within $1 \mathrm{~h}$ on the same track depends on the accuracy of the train localization system. The more accurate the train positions are known, the higher the throughput can be. However, an improvement of the localization accuracy of infrastructure-based localization systems requires additional infrastructure elements, that is, additional balises and axle counters, which increases the expenses even more.

Since the number of trains which are operated on a railway network is necessarily by orders of magnitudes smaller than the number of track segments in the network, an immense reduction of costs could be achieved by substituting the infrastructure-based train localization system by an on-board train localization system. Several approaches for train-borne localization have been proposed in recent years $[3,4,5,6,7,8,9,10]$. All of these approaches share a sensor setup that combines at least satellite navigation (GNSS) with a velocity sensor and a digital track map (cf. Fig. 1). Various GNSS systems like GPS, GLONASS, and Galileo with and without satellite-based augmentation systems (SBAS) and several velocity sensors, for exam- 


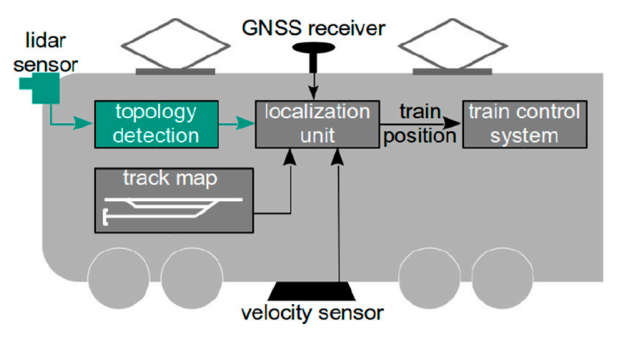

Figure 1: Extended setup for train-borne localization systems as described in [13]. Gray boxes indicate traditional elements of train-borne localization while the green boxes indicate the novel contribution of this paper.

ple, odometers, Doppler radars, optical sensors like HaslerRail CORRail 1000 or the eddy current sensor $[11,5,12]$ have been used in various combinations.

However, systems that only use GNSS, velocity sensors, and a digital track map suffer from two facts. First of all, GNSS is not available everywhere. For example, in tunnels, under bridges, in station concourses, and in thick forests, GNSS systems cannot provide valid position measurements. Moreover, even if a GNSS receiver receives enough signals to calculate a position it might be biased or even invalid due to multipath effects or GNSS jamming and spoofing. A failure of the GNSS sensor cannot be completely compensated by the velocity sensor and the digital track map. The velocity sensor can only be used to determine the distance traveled by the train but cannot decide which branch at a turnout is taken by the train. Therefore, the position measurement becomes ambiguous at turnouts. Therefore, a trackselective, reliable train-positioning system requires an additional device to determine on which track the train is currently located or which branching track the train is taking at a turnout [14].

Besides track selectivity, a train positioning system has to be able to compensate drift of the velocity sensor and operate even when GNSS is unavailable. This can be done using landmarks along the track. Besides others, level crossings [7], platforms [15], bridges and tunnels [6], masts of the overhead wiring as well as turnouts again can serve as landmarks. Our analysis of different sensor concepts discussed in [15] has shown that lidar (light detection and ranging) sensors have the largest potential to fill the remaining sensory gap for train-borne localization. Compared with camera systems, lidar sensors are independent of external (natural or artificial) illumination so that they can be operated every time and everywhere. The measuring volume of an appropriate lidar sensor is large enough to sense the ego track, the neighboring tracks, and objects next to the tracks like the above-mentioned landmarks. Therefore, this article investigates in which way lidar sensors can be used to detect track elements (Sections 2 and 3) and shows experimental results with a prototype system for a light rail scenario (Sections 4 and 5).

\section{REQUIREMENTS ON LIDAR SENSORS AND MARKET REVIEW}

Lidar sensors are optical devices. They exist in a variety of realizations that differ amongst others in the number of degrees of freedom. Simple laser distance sensors used by craftsmen are one-dimensional and measure the distance of a specific object directly. The rotation of one laser beam in two directions enables a full three-dimensional measurement of all points in a spherical environment what is used for example in surveying and mapping applications. This article considers sensors rotating in one direction perpendicular to the propagation 
direction of the laser beam. They are often referred to as single-layer sensors. Thereby, several hundred measurements in a two-dimensional environment can be obtained one after another. All measurement points within one revolution are called a scan. Thus, lidar sensors can provide a height profile of the environment containing also rails, tracks, and turnouts.

Relevant lidar sensor properties for detecting those infrastructure elements are angular resolution, measurement rate, field of view, and range. These will be described in detail assuming a sensor with one vertical scan layer mounted on a train as shown in Fig. 2. Further properties, such as the spot size and beam divergence, or the combination of several lidar sensors go beyond the scope of this article and have been discussed in [15].

As the minimal distance between most tracks is $3.5 \mathrm{~m}$, the lidar sensor should cover at least this distance plus half of the standard gauge of $1.435 \mathrm{~m}$ on straight tracks. Considering also the deflection of the train in curves, the measurement width on ground $W$ should be at least 6 $\mathrm{m}$ to the left as well as to the right side. Even when mounting the sensor on top of a train (height $H \approx 3.5 \mathrm{~m}$ ), the field of view has to be $\alpha \geq 120^{\circ}$ to reach $W=6 \mathrm{~m}$ (cf. Fig. $2 \mathrm{~b}$ ).

The range describes the interval of the distance in which the sensor is capable of scanning objects. For our purpose, distances up to $10 \mathrm{~m}$ at a remission of about $10 \%$ are sufficient. The range is often given for $100 \%$ remission (ideal reflector). But neither ballast nor rusted rails have this property.

The most relevant property is the angular resolution $\Delta \varphi$ (cf. Fig. 2b). It influences the discretization of objects in lateral direction (cf. [15, Fig. 3a]). Thus, it should be as small as possible. To ensure a reliable detection of objects, they should be hit by at least three beams [16]. In a height of $H=3.5 \mathrm{~m}$ with $\Delta \varphi=0.5^{\circ}$ and with negligible spot size, the minimal object size at a width on ground of $W=6 \mathrm{~m}$ is $23 \mathrm{~cm}$. With $\Delta \varphi=0.1^{\circ}$, it is only $5 \mathrm{~cm}$, which makes it still difficult to detect, for example, the profile of grooved rails on level crossings.

Besides those properties that already influence the potentials of the lidar sensor when the train stands still, scans spread widely when the train has a nonzero velocity $v$. The measurement rate $f$ determines the number of scans per second. The larger the measurement rate and the smaller the train velocity are, the better the objects are discretized along the track (cf. [15, Fig. 3b]). For example, objects smaller than $2.75 \mathrm{~m}$ may be missed at a rate of 30 scans per second, driving with a velocity of $100 \mathrm{~km} / \mathrm{h}$, and considering that an object can only be recognized if it was hit by three scans, again. Furthermore, with a higher measurement rate it is easier to drop erroneous measurements [16].

Our requirements on lidar sensors are summarized in Table 1. Table 2 gives a comprehensive overview on vendors and models, which meet most requirements. If a vendor offers several sensors, the most promising ones are selected. All those sensors use the time-of-flight method to measure the distance of an object. They have a laser beam with a wavelength of $905 \mathrm{~nm}$ and are laser class 1. Due to our restrictions on angular resolution and measurement
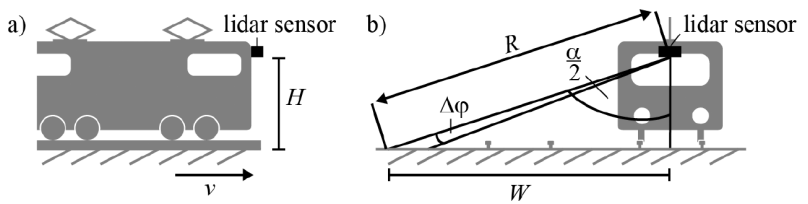

Figure 2: Single-layer lidar sensor on a train: (a) side view and (b) front view (height $H$, velocity of the train $v$, width on ground $W$, range $R$, field of view $\alpha$, angular resolution $\Delta \varphi$ ). 
Table 1: Primary requirements on lidar sensors for detecting railway infrastructure.

\begin{tabular}{|c|c|c|}
\hline Property (unit) & Minimal value & Optimal value \\
\hline Angular resolution $\Delta \varphi$ (deg.) & $<1 / 2$ & $<1 / 10$ \\
\hline Measurement rate $f$ (scans/s) & $\geq 30$ & $\geq 50$ \\
\hline Field of view $\alpha$ (deg.) & $\geq 120$ & $\geq 180$ \\
\hline Range $R(\mathrm{~m})$ & \multicolumn{2}{|c|}{$0.2-10.0$} \\
\hline Price (EUR) & \multicolumn{2}{|c|}{$\leq 10,000$} \\
\hline
\end{tabular}

rate, multilayer sensors used primarily for driver assistance (e. g., ibeo LUX) and autonomous driving (e. g., Velodyne lidar) have not been surveyed. Our price limit precludes high-end sensors established in the railway domain (e. g., Riegl VQ-450 or High Speed Profiler of Fraunhofer IPM) or in mapping [17]. They are usually several times more expensive than the price level admissible for the whole train localization system.

Based on our requirement analysis, the Pepperl+Fuchs lidar sensor in Table 2 is the most appropriate device since it has by far the finest angular resolution and allows high measurement rates. The lidar sensor from the same vendor proposed in [15] is not sufficient since it has just $R_{\max }=3 \mathrm{~m}$.

\section{DETECTING RAILS, TRACKS, AND TURNOUTS IN LIDAR SENSOR DATA}

The results of the lidar sensor data processing can be used for deriving topological information. This complements both geographical and longitudinal position information (cf. Section 1 and Fig. 1). At first, Section 3.1 characterizes lidar sensor data. Then, Section 3.2 introduces some railway characteristics. As most of those railway infrastructure elements are formed by rails, Section 3.3 describes our rail detector. Finally, a concept for track and turnout detection is presented in Section 3.4.

\subsection{Characteristics of the lidar sensor data}

This article assumes that a lidar sensor delivers a set of valid measurement triples $(\varphi, D, E)$. The rotation angle $\varphi$ ranges in the interval $0^{\circ} \leq \varphi<360^{\circ}$ whereby $270^{\circ}$ is below the sensor, $0^{\circ}$ is on the right side, and $180^{\circ}$ is on the left side of the sensor when driving forwards. $\varphi$ is discretized equidistant with an angular resolution of $\Delta \varphi$. Both the radial distance $D$ and the echo $E$ are positive.

$D$ and $\varphi$ describe the contour of the environment in the $x-y$ plane with $x=D \cdot \cos (\varphi)$ and $y$ $=D \cdot \sin (\varphi)$. When adding motion information as $z$ value in forward direction of the train, a three-dimensional representation $(x, y, z)$ can be derived. Both the polar and the Euclidean representation are equivalent. For illustration purposes, the latter one is preferred.

Let us assume a horizontal, flat, and homogeneous ground measured from a lidar sensor mounted at $H=2.5 \mathrm{~m}$ with $\Delta \varphi=0.071^{\circ}$. The horizontal distance between neighboring measurements spreads widely for $270^{\circ}>\varphi>180^{\circ}$ and $270^{\circ}<\varphi<360^{\circ}$, respectively. Thus, the corresponding radial distances increase accordingly and form a concave shape with a minimum at $\varphi=270^{\circ}$. While the interval of $0 \mathrm{~m} \leq|x| \leq 0.5 \mathrm{~m}$ covers 158 measurement points, the interval of $5.5 \mathrm{~m} \leq|x| \leq 6 \mathrm{~m}$ holds only 25 . Since the angle of incidence of the laser beam decreases the larger $|x|$ is, the echo has a convex shape with its maximum at $\varphi=270^{\circ}$. 


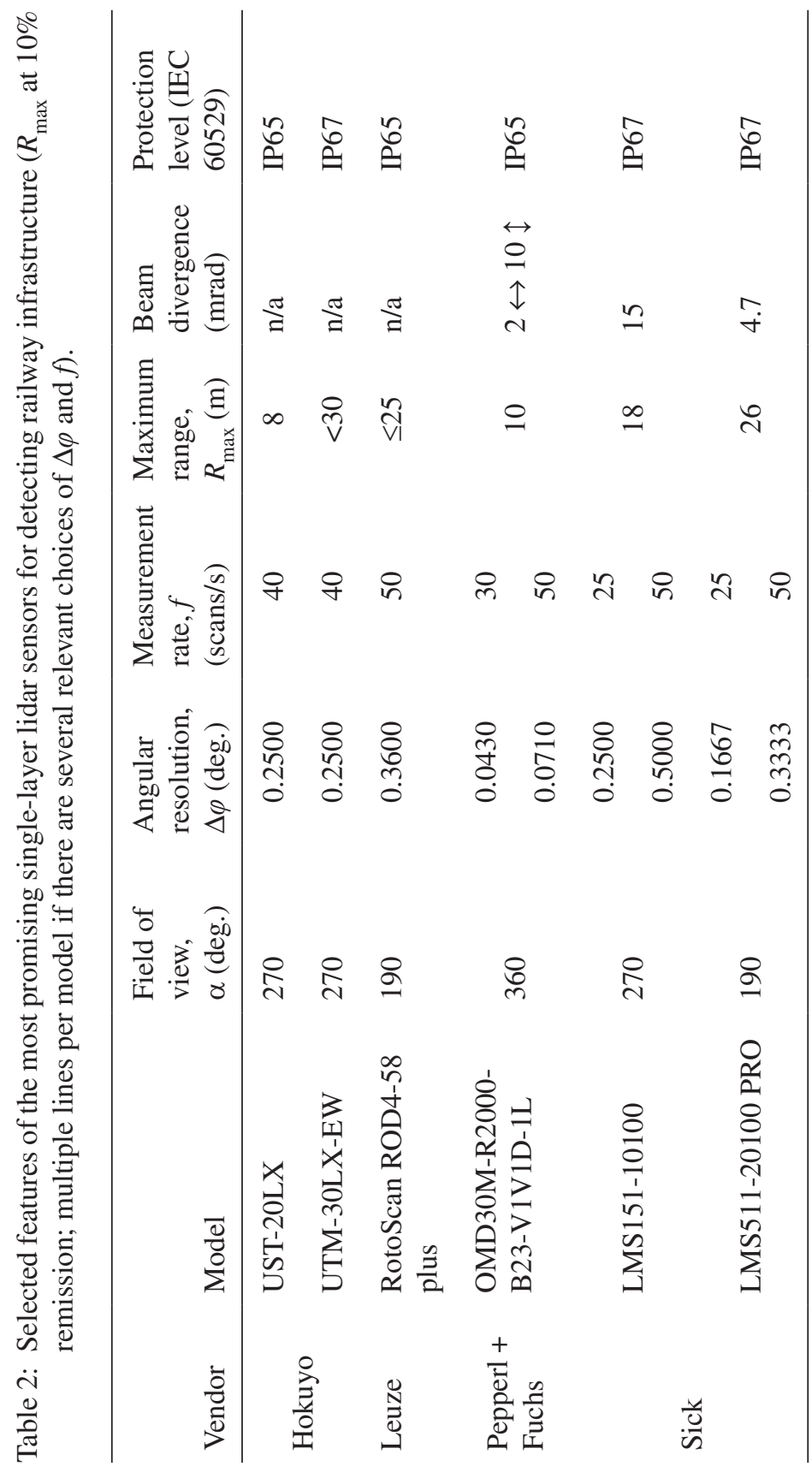




\subsection{Relevant railway characteristics}

This section describes features of relevant railway infrastructure elements that can be detected by lidar sensors. Our case study (cf. Section 4) will take place in a light rail scenario where grooved rails are typical (cf. left part of Fig. 4). Their profile has a groove depth $d_{\mathrm{g}}$, a groove width $w_{\mathrm{g}}$, and a rail width $w_{\mathrm{r}}$. Values for a typical 59R2 profile are $d_{\mathrm{g}} \approx 4.7 \mathrm{~cm}, w_{\mathrm{g}} \approx 4.4 \mathrm{~cm}$, and $w_{\mathrm{r}} \approx 11.3 \mathrm{~cm}$. Due to the design of turnouts, especially $w_{\mathrm{g}}$ becomes smaller near the frog and wider near the blade.

Tracks are formed by parallel pairs of rails. Their gauge $G$ varies within a small interval. Our scenario has standard gauge $G=1.435 \mathrm{~m}$ and a minimal lateral distance of parallel tracks of $3.05 \mathrm{~m}$.

\subsection{Rail detection}

The detection of rails in the lidar data is primarily based on distance measurements. Every scan is a discrete signal, which is equidistant over $\varphi$. Thus, $\varphi$ can be treated like the time in sequential signal processing. Low frequencies contain especially the ground. Grooved rails and measurement errors are part of the higher frequencies. Since the focus of our detection is on rails, every scan is normalized at first. The result of a zero-phase digital low-pass filtered scan [18] is subtracted from the original scan. The filter width is adapted for every $\varphi$ such that the filter always covers almost the same width in $x$ direction. All points are weighted equally using a rectangular filter mask. So, this normalized signal still contains relevant high frequent parts, but it has no longer a concave shape. The filter width has to hold $w_{\mathrm{f}, \mathrm{d}}>\sqrt{2} w_{g}$ so that even at passing the grooved rail diagonally it is not normalized out. Figure 3 shows the distance measurements before and after normalization on the left side.

Motivated by Rahmig et al. [7] and Blug et al. [16], a template-matching approach detects grooved rails in the normalized distance data. Therefore, the shape of the grooved rail is of interest. As they are located below their environment, they cause an increase in the radial distance. Hence, normalized distances larger than $0 \mathrm{~m}$ are searched. Furthermore, grooved rails have a certain width that is not always completely detectable. The laser beam hits the groove almost traversely, so that the inner side next to the lidar sensor is occluded (cf. left part of Fig. 4). To avoid wrong detections potentially interesting areas with a width less than $w_{\mathrm{g}} / 2$
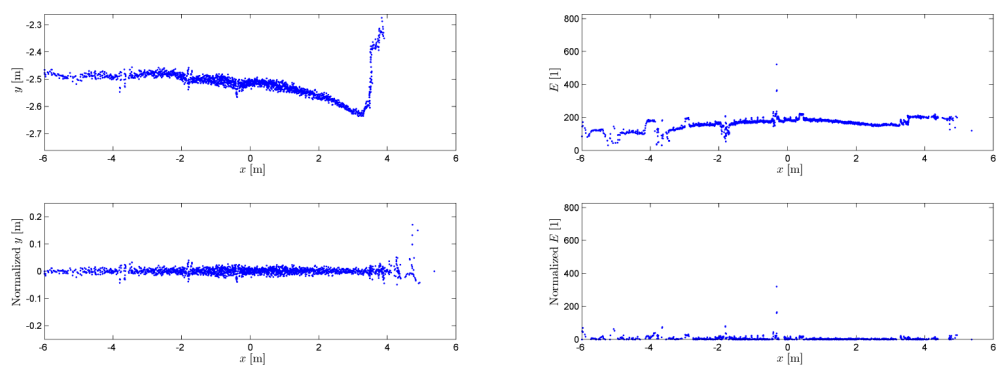

Figure 3: Normalization of lidar sensor data from a light rail scenario $(H=2.5 \mathrm{~m}, \Delta \varphi=$ $0.071^{\circ}$ ). Left: distance measurements before (above) and after normalization (below). Right: corresponding echo measurements before (above) and after normalization (below). 
are skipped. The same applies to areas with a mean depth of less than $2 \mathrm{~cm}$, which is less than $d_{\mathrm{g}} / 2$. Note that there is neither a reasonable maximum in depth nor in width. Especially at the blades grooved rails can contain holes (cf. [15, Fig. 4a]). The same applies to drain pipes that are located within the groove. In both cases, the measured groove depth is larger than $d_{\mathrm{g}}$. The measured groove width tends to infinity when a rail is passed in a flat angle. Since disturbances can hamper the detection of a groove at a glance finally, all regions that are not more than $\sqrt{ } 2 w_{\mathrm{g}}$ in the $x$ direction away from each other are merged. Exemplary results of the rail detection from distances are shown in the right upper plot in Fig. 4.

Although the echo value $E$ depends on a couple of parameters [19] and it is usually not calibrated, it allows to distinguish between different materials. The echo is also treated as an equidistant discrete signal with sampling interval $\Delta \varphi$ and the normalization approach is applied, again. The filter width has to be chosen larger, since diverse parts of the rail surface reflect the laser beam differently. Therefore, it has to hold $w_{\mathrm{f}, \mathrm{e}}>\sqrt{ } 2 w_{\mathrm{r}}$ to cover at least the whole grooved rail when passing it diagonally. Figure 3 shows echo measurements before and after normalization on the right side.

Changes in the material (such as bitumen, rusted, or blank steel) and the angle of incidence near and within grooved rails cause significant changes of the echo amplitude, while homogeneous areas are quite smooth. To detect grooved rails in the normalized echo their higher variance is in our focus. As the echo changes positively and negatively, the absolute value of the normalized echo is used. For every rotation angle, it is checked whether this is beyond a threshold $e_{t}$, then it is marked as a rail candidate. $e_{t}$ is chosen as twice the standard deviation of the absolute value of the normalized echo in the current scan. A normal distribution of the echo is assumed so that less than $5 \%$ of all values are above $e_{t}$. Five percentage equals the ratio of the width of typically two parallel tracks in light rail scenarios, that is, $4 w_{\mathrm{r}}$, and the target width of $2 W$. After applying this threshold, all regions that are not more than $\sqrt{2} w_{\mathrm{g}}$ in $x$ direction away from the other are merged, since significant changes of the echo may not be inside the groove. Finally, all regions that are smaller than $w_{\mathrm{g}}$ are skipped since they are too small for a grooved rail. Again, there is no reasonable maximum for the width of a region. Exemplary results of the rail detection from echoes are shown in the right lower plot in Fig. 4.
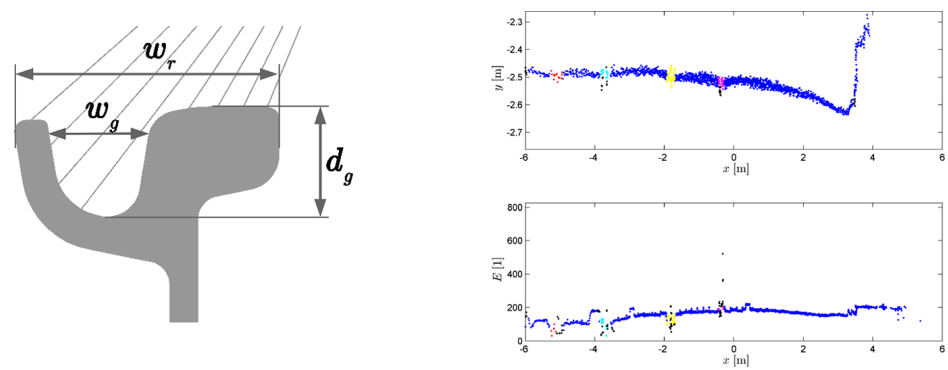

Figure 4: Left: grooved rail and its measurement from the upper right side with a lidar sensor (width of groove $w_{\mathrm{g}}$, depth of groove $d_{\mathrm{g}}$, and width of rail $w_{\mathrm{r}}$ ). Right: grooved rail detection in distance (above) and echo measurements (below). Manually labeled rails (ground truth) are shown in yellow and magenta (ego track) and in red and cyan (parallel track). Our rail detection results are shown in black and partially occlude the labeled points. All other distance or echo points are marked in blue and are neither detected as rail nor labeled as a rail area $\left(H=2.5 \mathrm{~m}, \Delta \varphi=0.071^{\circ}\right)$. 
As explained before, the echo can be just an indicator for potential rails. For example, road markings that are typical for a light rail scenario also have an increased echo, but are quite constant. The same applies for the distance where concave-shaped objects such as water passages can be similar to grooved rails. Therefore, the results of rail detection in distance and echo have to be combined. Therefore, an all-or-nothing approach is proposed. A region is only a potential rail when it is detected both in distance and echo and has at least a width of $w_{\mathrm{g}} / 2$

\subsection{Concept for track and turnout detection and integration in a train-borne localization system}

Detected rails (cf. Section 3.3) with a lateral distance of at least $d_{1}$ are searched since they might form a track. $d_{1}$ is a rough estimate that never underestimates $G-\varepsilon$, whereby $\varepsilon$ models the uncertainty in both the position of the rail detection and the gauge. It will be only some centimeters. Considering also at least three detections of the rail in the direction of travel and tracking our detections over time, one will see that tracks are converging into ( $d_{1}$ decreasing) or diverging from the ego track ( $d_{1}$ increasing). This happens only near turnouts or crossings. If one track splits into two or more tracks and those tracks are diverging, the train passed a turnout in facing direction. The train passed a turnout trailing if there were two tracks, the lateral distance of those was decreasing over time and finally became zero. Therefore, both the turnout and the branching direction thereon can be determined.

An alternative is the detection of parts of a turnout, for example, blade, frog, and guard rails, by their characteristic features. This is done for example in [19] for Vignole rails. The turnout and the branching direction can be derived again when taking into account their chronological sequence.

Additional information can be derived by comparing $d_{l}$ over time again. For parallel tracks, $d_{l}$ is almost constant. Therefore, one can also reason on which side the parallel tracks are located and compare this topological information with the geometry and topology of the railway network which is stored in the digital track map (cf. green parts in Fig. 1). Thus, position ambiguities can be significantly reduced or even avoided. The same applies for the detection of the beginning and end of level crossings [7], bridges and tunnels [6], or platforms [15].

\section{CASE STUDY IN A LIGHT RAIL SCENARIO}

To perform test drives in a light rail scenario in the city of Karlsruhe, the lidar sensor was mounted at the tail of a road vehicle (cf. left part of Fig. 5). This setup enables the validation of our concept from Section 3 without requiring a special train and avoids the respective expenses. The missing directional stability resembles the hunting oscillation of a train.

A prototype of the Pepperl+Fuchs OMD30M-R2000-B23-V1V1D-1L lidar sensor has been used for our case study. Compared with the sensor used in [15], this sensor has an unlimited field of view, a smaller angular resolution, and a higher measurement rate. In addition, it delivers distance and echo information at the same time. The distance measurements $D$ are given in meter as fixed point numbers with a resolution of $1 \mathrm{~mm}$. Echo values are integers in the interval of $32 \leq E \leq 4095$ (cf. Section 3.1). $f=50$ scans $/ \mathrm{s}, \Delta \varphi=0.071^{\circ}$ (cf. Table 2), and $H=2.5 \mathrm{~m}$ (cf. Fig. 2) were chosen.

Our data cover several kilometers of tracks measured at different times of the day. Selected data sets are described in Table 3. Figure 5 shows the passing of a turnout trailing in the right part. Finally, manually labeled rails in those data sets are used as ground truth to evaluate our detection results (cf. the right part of Fig. 6 where every labeled rail has a different color). 

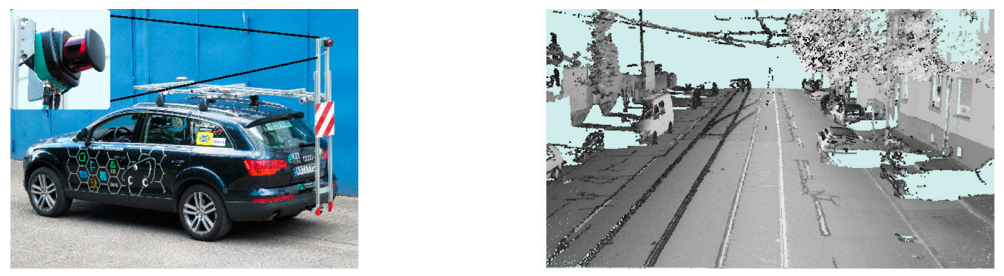

Figure 5: Left: setup for a light rail scenario with the lidar sensor enhanced on the upper left side. Right: three-dimensional view of the lidar sensor measurements from data set T1 when passing a turnout with grooved rails and standard gauge trailing. The echo measurements are mapped to gray values (mean velocity of the vehicle $\bar{v}=3.9 \mathrm{~m} / \mathrm{s}$ ).

Table 3: Brief description of the measurements on May 16, 2014, obtained in the city of Karlsruhe.

\begin{tabular}{lllllllll}
\hline $\begin{array}{l}\text { Data } \\
\text { set }\end{array}$ & $\begin{array}{l}\text { Number } \\
\text { of scans }\end{array}$ & $\begin{array}{l}v_{\min } \\
(\mathrm{m} / \mathrm{s})\end{array}$ & $\begin{array}{l}v_{\max } \\
(\mathrm{m} / \mathrm{s})\end{array}$ & $\begin{array}{l}\bar{v} \\
(\mathrm{~m} / \mathrm{s})\end{array}$ & $\begin{array}{l}\text { Length } \\
(\mathrm{m})\end{array}$ & $\begin{array}{l}\text { Number } \\
\text { of rails }\end{array}$ & $\begin{array}{l}\text { Number of } \\
\text { turnouts }\end{array}$ & $\begin{array}{l}\text { Branching } \\
\text { direction on } \\
\text { turnouts }\end{array}$ \\
\hline $\mathrm{P} 1$ & 1517 & 4.9 & 7.1 & 6.7 & 207.9 & 4 & 0 & $\mathrm{n} / \mathrm{a}$ \\
$\mathrm{P} 2$ & 999 & 0.4 & 6.4 & 4.8 & 95.1 & 4 & 0 & $\mathrm{n} / \mathrm{a}$ \\
$\mathrm{P} 3$ & 2507 & 0.7 & 10.5 & 7.7 & 397.2 & 4 & 0 & $\mathrm{n} / \mathrm{a}$ \\
$\mathrm{T} 1$ & 704 & 3.7 & 4.1 & 3.9 & 54.4 & $4-6$ & 2 & Trailing left \\
$\mathrm{T} 2$ & 3472 & 0 & 4.6 & 3.0 & 214.7 & $2-4$ & 2 & Trailing right, \\
& & & & & & & & facing right \\
$\mathrm{N}$ & 2649 & 0 & 13.3 & 6.6 & 355.9 & 0 & 0 & n/a \\
\hline
\end{tabular}

Note: Scans are measured with the Pepperl+Fuchs OMD30M-R2000-B23-V1V1D-1L lidar sensor. Velocities and lateral distances of the vehicle are measured with an OXTS RT3000 for documentation purposes (minimum, maximum, and mean velocity $v_{\min }, v_{\max }$, and $\bar{v}$ ).
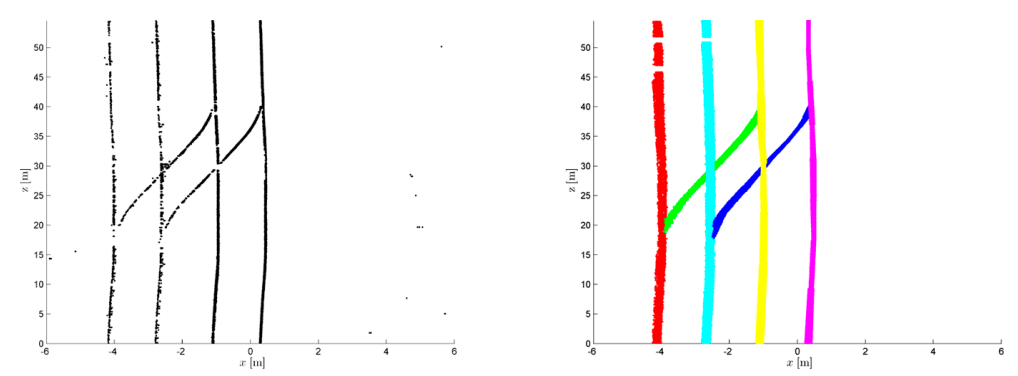

Figure 6: Left: top view on rail detections ( $D$ and $E$ combined) in data set T1 when passing a turnout trailing with grooved rails and standard gauge. Right: top view on manually labeled rails in data set T1 (ground truth; one color per rail). 
Our application area lies in the interval of $|x| \leq 6 \mathrm{~m}$. Thus, only rotation angles in the interval of $202^{\circ} \leq \varphi \leq 338^{\circ}$ are considered. Other objects in the environment, such as trains on a parallel track, hamper a measurement behind them. They are detected depending on their height and slope. For the former one, it is checked whether the current $y$ value is too high $(y>-H+0.25 \mathrm{~m})$. For the latter, it is tested whether the slope in the $x-y$ plane over 15 consecutive rotation angles is at least 5 . An additional boundary is set on their inner side to prevent the evaluation of the data behind them. Finally, the missing or skipped data within these boundaries are padded for both distance and echo values. Thereby, missing parts between valid measurements have been interpolated linearly in the angular representation. Missing data at the boundaries are filled with the next valid measurement.

\section{EXPERIMENTAL RESULTS}

At first, the influence of $w_{\mathrm{f}, \mathrm{d}}$ and $w_{\mathrm{f}, \mathrm{e}}$ on the rail detections is examined. Therefore, the detection results are divided into four categories. The number of correctly detected rails is denoted with $t_{\mathrm{p}}$. If a rail is detected although there is non in the data, it is counted as $f_{\mathrm{p}}$. The same applies for correct detections of everything but not a rail $\left(t_{n}\right)$ and not detected rails although they appear within the data $\left(f_{n}\right)$. The true positive rate $f_{\text {tp }}$ and the false positive rate $f_{\mathrm{fp}}$ are thus defined by

$$
f_{t p}=\frac{t_{p}}{t_{p}+f_{n}} \quad \text { and } \quad f_{f p}=\frac{f_{p}}{t_{n}+f_{p}}
$$

Every scan is divided into regions to calculate $f_{\mathrm{tp}}$ and $f_{\mathrm{fp}}$. In the scan shown in the upper right plot of Fig. 4 exist four regions of rails (red, cyan, yellow, and magenta) and five regions of everything else (five times blue). The rails in the cyan and magenta region in the distance measurements have been detected $\left(t_{\mathrm{p}}=2\right)$, but not the rails in the red and yellow regions $\left(f_{n}=2\right)$. As also two rails near the borders $(x \approx-6 \mathrm{~m}$ and $3 \mathrm{~m}<x<4 \mathrm{~m})$ are detected, it holds $f_{\mathrm{p}}=2$ and $t_{n}=3$.

To find optimum filter widths, 100 different values have been evaluated on data set P1 in MATLAB. Some results are shown in Table 4. A high true positive rate is of primary interest for rail detection, since false positives can be filtered out afterwards, but missing detections cannot be reconstructed. Considering also the computational effort $w_{\mathrm{f}, \mathrm{d}}=30 \mathrm{~cm}$ is chosen,

Table 4: Influence of the filter widths on the detection results on data set P1.

\begin{tabular}{lllllllllll}
\hline$w_{\text {f.d }}(\mathrm{cm})$ & 10 & 20 & 30 & 40 & 50 & 60 & 70 & 80 & 90 & 100 \\
\hline$f_{\text {tp }, \mathrm{d}}(\%)$ & 22.60 & 63.32 & 73.96 & 75.93 & 76.05 & 75.34 & 73.60 & 71.54 & 68.82 & 65.65 \\
$f_{\text {fp.d }}(\%)$ & 5.07 & 10.93 & 16.32 & 20.56 & 23.87 & 25.65 & 26.34 & 26.49 & 26.27 & 25.56 \\
\hline$w_{\text {f.e }}(\mathrm{cm})$ & 10 & 20 & 30 & 40 & 50 & 60 & 70 & 80 & 90 & 100 \\
\hline$f_{\text {tp }, \mathrm{e}}(\%)$ & 85.19 & 86.08 & 87.09 & 87.69 & 88.14 & 88.79 & 89.00 & 89.29 & 89.77 & 89.95 \\
$f_{\text {tp.e }}(\%)$ & 38.86 & 42.89 & 45.44 & 45.89 & 46.38 & 47.49 & 48.68 & 49.59 & 50.15 & 50.58 \\
\hline
\end{tabular}

Note: True positive rate $f_{\mathrm{tp}}$ and false positive rate $f_{\mathrm{fp}}$ are calculated on distance measurements for $w_{\mathrm{f}, \mathrm{d}}$ and on echo measurements for $w_{\mathrm{f}, \mathrm{e}}$. 
since the gradient of $f_{\mathrm{tp}, \mathrm{d}}$ decreases for larger $w_{\mathrm{f}, \mathrm{d}}$. The echo filter width $w_{\mathrm{f}, \mathrm{e}}=40 \mathrm{~cm}$ is chosen on the plateau in $f_{\mathrm{fp}, \mathrm{e}}$.

These parameters are used to evaluate all data sets described in Table 3. Table 5 shows quantitative results. Considering all data sets except $\mathrm{N}$, the rail detections in the distance measurements are good (quite low $f_{\mathrm{fp}, \mathrm{D}}$ ). The detections in the echo measurements contain almost every grooved rail independent of whether turnouts occur or not $\left(\operatorname{high} f_{\text {tp. }}\right)$. The rates are sometimes even better compared to the training data set P1. However, $f_{\mathrm{fp}, \mathrm{E}}$ " $50 \%$ is not acceptable, so the detections must not be based solely on echo measurements. Thus, a rigid all-or-nothing approach is proposed in Section 3.3 that reduces the number of misdetections significantly (cf. left part of Fig. 6).

The ego track is correctly detected in the left part of Fig. 6, although due to guidance reasons the width of the right rail is smaller than expected $(0 \mathrm{~m}<x<1 \mathrm{~m}, 25 \mathrm{~m} \leq z<35 \mathrm{~m})$. The minimum admissible width of $w_{g} \approx 3 \mathrm{~cm}$ makes the demanding grooved rail detection even harder. However, almost every rail of the ego track is detected $\left(f_{\mathrm{tp}, \mathrm{C}}=79.09 \%\right)$. Some rails near the frog $(x \approx-1 \mathrm{~m}, 25 \mathrm{~m}<z<30 \mathrm{~m})$ and at the blade $(-1 \mathrm{~m}<x<1 \mathrm{~m}, 35 \mathrm{~m}<z<40 \mathrm{~m})$ are not detected, since they might be too close to each other which results in $f_{\mathrm{tp}, \mathrm{C}}=43.28 \%$ on the branching track with green and blue rails. However, even the parallel track $(x<-2 \mathrm{~m})$ is partially detected with $f_{\text {tp }, \mathrm{C}}=11.29 \%$ for the red and cyan rails. There are only few conspicuous false positives near the curbs $(x>3 \mathrm{~m})$, which leads to $f_{\mathrm{fp}, \mathrm{C}}<3 \%$ on the whole data set.

Finally, data set $\mathrm{N}$ is considered. Since it contains no rails, true positive rates cannot be calculated. Contrary, the false-positive rate seems to be quite bad. But, as there is just one region per scan for everything but a rail (and none for rails), every incorrect rail detection scores. $f_{\mathrm{fp} . \mathrm{C}} \approx 18 \%$ states that our all-or-nothing approach had incorrect detections just in every sixth scan and is thus much better than the detections in either distance or echo measurements.

The results of this case study prove that the sensor resolution is sufficient for railway infrastructure detection. This is noticeable since the recognition of grooved rails with their small width of $<5 \mathrm{~cm}$ and partial occlusion is much more difficult compared to that of Vignole rails with a height of at least $13 \mathrm{~cm}$. A measurement rate of $50 \mathrm{scans} / \mathrm{s}$ and an ego

Table 5: Results of the rail detection in distance $D$ and/or echo $E$ measurements (true positive rate $f_{\mathrm{tp}}$ and false positive rate $f_{\mathrm{fp}}$ ).

\begin{tabular}{lllllll}
\hline & \multicolumn{2}{c}{$D$ only } & \multicolumn{2}{c}{$E$ only } & \multicolumn{2}{c}{$D$ and $E$ combined } \\
\cline { 2 - 7 } Data set & $f_{\mathrm{tp}, D}(\%)$ & $f_{\mathrm{fp}, \mathrm{D}}(\%)$ & $f_{\mathrm{tp}, \mathrm{E}}(\%)$ & $f_{\mathrm{fp}, \mathrm{E}}(\%)$ & $f_{\mathrm{tp}, \mathrm{C}}(\%)$ & $f_{\mathrm{fp}, \mathrm{C}}(\%)$ \\
\hline $\mathrm{P} 1$ & 73.96 & 16.32 & 87.69 & 45.89 & 39.33 & 4.90 \\
$\mathrm{P} 2$ & 81.28 & 19.99 & 99.68 & 70.56 & 51.04 & 4.46 \\
$\mathrm{P} 3$ & 73.18 & 11.53 & 97.76 & 81.27 & 40.66 & 2.56 \\
$\mathrm{~T} 1$ & 74.55 & 11.80 & 91.58 & 56.26 & 45.31 & 2.36 \\
$\mathrm{~T} 2$ & 72.45 & 16.66 & 98.48 & 81.45 & 60.28 & 9.29 \\
$N$ & $\mathrm{n} / \mathrm{a}$ & 43.07 & $\mathrm{n} / \mathrm{a}$ & 100.0 & $\mathrm{n} / \mathrm{a}$ & 17.63 \\
\hline
\end{tabular}


track detection rate of about $80 \%$ leads already to information on tracks with a rate of about $40 \mathrm{~Hz}$. When considering also the past detections in a tracking approach those rates might even be improved. In addition, neighboring and branching tracks are already detected and thus deliver additional topological information.

\section{CONCLUSIONS}

Accurate, track-selective train-borne localization systems are a key component in future train protection systems. While previously proposed systems heavily depend on the availability of GNSS measurements, a robust solution requires sensors that are diverse enough to compensate GNSS failures. The possibility to detect turnouts on the track and the branching direction at turnouts has been identified as the key feature of a robust localization system. It has been shown that lidar sensors are able to fill this sensory gap and that they are able to provide the previously missing information.

A lidar sensor provides in each scan a very accurate distance profile of the environment of the train. This profile can be used to detect railway infrastructure elements like individual rails, tracks, and turnouts, even in the hard case of grooved rails that often occur in light rail systems and on level crossings. However, the set of recognizable objects is even larger and includes parallel tracks, platforms, and masts of the overhead wiring, among others. Those objects can be used in future systems as additional landmarks to increase the localization accuracy especially in longitudinal direction. Moreover, the bearing and orientation of other tracks can be compared to the topology of the railway network stored in the digital map. Thus, it reduces the number of position hypothesis and supports lateral localization. The key idea to detect relevant objects is to perform template matching between the distance profile that was sensed by the lidar sensor and template profiles for the infrastructure elements. This step benefits from the fact that almost all infrastructure elements in the railway environment are standardized, that is, the appearance of those objects shows only little variation.

The theoretical findings and arguments of our approach are supported by our experimental results. Based on a light rail setup, the ability of grooved rails detection has been investigated. The task of detecting grooved rails with a lidar sensor is much more difficult than detecting a standard Vignole rail. Vignole rails are raised objects and have thus a very distinctive profile in a lidar scan while for grooved rails only the groove itself can be sensed by a lidar sensor. However, our experimental results show that even this difficult task can be solved with the template-matching approach.

Our next steps in the development of lidar-based train localization will be to extend the template-matching approach to the other objects that can be used as landmarks for localization and to develop a sensor fusion concept that takes account of the different properties, failure modes, accuracies, and availabilities of a diverse sensor setup in a train localization system.

\section{ACKNOWLEDGMENT}

The authors thank R. Rössling and J.-R. Krato from Pepperl+Fuchs for providing us prototypes and knowledge on their sensors, and J. Ziegler for supporting the software development.

\section{REFERENCES}

[1] Theeg, G. \& Vlasenko, S. (eds.), Railway Signalling \& Interlocking: International Compendium, Eurailpress, 2009. 
[2] Stanley, P. (ed.), ETCS for engineers, Eurailpress, 2011.

[3] Albanese, A., Labbiento, G., Marradi, L. \& Venturi, G., The RUNE project: the integrity performances of GNSS-based railway user navigation equipment, Proc. Joint Railway Conf., pp. 211-218, 2005. doi: http://dx.doi.org/10.1109/rrcon.2005.186082

[4] Jiang, Z., Digital route model aided integrated satellite navigation and low-cost inertial sensors for high-performance positioning on the railways, PhD thesis, University College London, 2010.

[5] Hensel, S., Hasberg, C. \& Stiller, C., Probabilistic rail vehicle localization with eddy current sensors in topological maps. IEEE Trans. of Intell. Transp. Syst., 12(4), pp. 1525-1536, 2011. doi: http://dx.doi.org/10.1109/TITS.2011.2161291

[6] Rahmig, C., Lüddecke, K. \& Lemmer, K., Tunnels and bridges as observable landmarks within a modified multi-hypothesis based map-matching algorithm for train positioning. Proc. European Navigation Conf., 2012.

[7] Rahmig, C., Johannes, L. \& Lüddecke, K., Detecting track events with a laser scanner for using within a modified multi-hypothesis based map-matching algorithm for train positioning, Proc. European Navigation Conf., 2013.

[8] Heirich, O., Robertson, P. \& Strang, T., RailSLAM - localization of rail vehicles and mapping of geometric railway tracks, Proc. IEEE Int. Conf. Robotics and Automation, pp. 5192-5199, 2013. doi: http://dx.doi.org/10.1109/icra.2013.6631322

[9] Albrecht, T., Lüddecke, K. \& Zimmermann, J., A precise and reliable train positioning system and its use for automation of train operation, Proc. IEEE Int. Conf. on Intell. Rail Transp., pp. 134-139, 2013. doi: http://dx.doi.org/10.1109/icirt.2013.6696282

[10] Lauer, M. \& Stein, D., Algorithms and concepts for an onboard train localization system for safety-relevant services, Proc. IEEE Int. Conf. Intell. Rail Transp., pp. 65-70, 2013. doi: http://dx.doi.org/10.1109/icirt.2013.6696269

[11] Engelberg, T. \& Mesch, F., Eddy current sensor system for non-contact speed and distance measurement of rail vehicles. Computers in Railways VII, eds. J. Allan, R. Hill, C. Brebbia, G. Sciutto \& S. Sone, WIT Press: Southampton, pp. 1261-1270, 2000.

[12] Lauer, M. \& Stein, D., A train localization algorithm for train protection systems of the future. IEEE Trans. Intell. Transp. Syst., 16(2), pp. 970-979, 2015.

[13] Stein, D., Spindler, M. \& Lauer, M., Lidar sensors for detecting railway infrastructure and their usage in train-borne localization systems, Poster: MoLaS Technology Workshop, Freiburg, 2014.

[14] Mesch, F., Puente León, F. \& Engelberg, T., Train-based location by detecting rail switches. Computers in Railways VII, eds. J. Allan, R. Hill, C. Brebbia, G. Sciutto \& S. Sone, WIT Press: Southampton, pp. 1251-1260, 2000.

[15] Stein, D., Lauer, M. \& Spindler, M., An analysis of different sensors for turnout detection for train-borne localization systems. Computers in Railways XIV: Railway Engineering Design and Optimization, eds. C. Brebbia, N. Tomii, P. Tzieropoulos \& J. Mera, WIT Press: Southampton, WIT Transactions on The Built Environment, vol. 135, pp. 827-838, 2014.

[16] Blug, A., Baulig, C., Wölfelschneider, H. \& Höfler, H., Fast fiber coupled clearance profile scanner using real time 3D data processing with automatic rail detection, Proc. IEEE Intell. Veh. Symp., pp. 658-663, 2004. doi: http://dx.doi.org/10.1109/ ivs.2004.1336462

[17] Kern, F., Marktübersicht Terrestrische Laserscanner (TLS) (market survey terrestrial laser scanning (TLS)) - Version 9, 2010. 
[18] Oppenheim, A.V., Schafer, R.W. \& Buck, J.R., Discrete-Time Signal Processing, 2nd edn., Prentice Hall, 1999.

[19] Hackel, T., Stein, D., Maindorfer, I., Lauer, M. \& Reiterer, A., Track detection in 3D laser scanning data of railway infrastructure, Proc. IEEE Int. Instrumentation and Measurement Technology Conf., pp. 693-698, 2015. doi: http://dx.doi.org/10.1109/ i2mtc.2015.7151352 